UPDATE ARTICLE

ARTIGO DE ATUALIZAÇÃO

\section{New treatment approaches for relapsing/refractory Hodgkin's lymphoma: An overview of the Brazilian scenario}

\author{
Novas abordagens de tratamento para o linfoma de \\ Hodgkin recidivante/refratário: uma revisão \\ Guilherme Fleury Perini', Valeria Buccheri², Talita Bueno da Silveira ${ }^{3}$, \\ Adriana Marques Damasco Penna ${ }^{4}$, Juliano Julio Cerci ${ }^{5}$, Carlos Eduardo Bacchi ${ }^{6}$, \\ Luciana Fanti', Tânia Barreto ${ }^{8}$
}

DOI: 10.21115/JBES.v10.n2.p190-197

\section{Keywords:}

Hodgkin's lymphoma, unmet medical needs, treatments

\section{Palavras-chave:}

linfoma de Hodgkin, necessidades médicas não atendidas, tratamentos

\begin{abstract}
Hodgkin's lymphoma $(\mathrm{HL})$ is a B-cell malignancy with a classical bimodal distribution with incidence peaking in the third and sixth decades of life. The purpose of this review is to describe the current unmet medical need for relapsing/refractory $\mathrm{HL}$ and the main data of emerging treatments, including brentuximab vedotin, the immune checkpoint inhibitors nivolumab and pembrolizumab, as well as other compounds in development. Available guidelines for relapsing/refractory HL are discussed.
\end{abstract}

\section{RESUMO}

O linfoma de Hodgkin (LH) é uma neoplasia de células B com distribuição bimodal clássica com pico de incidência na terceira e sexta décadas de vida. O objetivo desta revisão é descrever as atuais necessidades médicas não atendidas dos pacientes com LH recidivante/refratário e os dados principais dos tratamentos emergentes, incluindo brentuximabe vedotina, os inibidores do ponto de verificação imunológico, nivolumabe e pembrolizumabe, bem como outros compostos em desenvolvimento. Além disso, discutem-se as diretrizes disponíveis para LH recidivante/refratário.

\footnotetext{
Received on: 08/05/2018. Approved for publication on: 10/07/2018

1. Hospital Albert Einstein, São Paulo, SP, Brazil.

2. Instituto do Câncer do Estado de São Paulo (Icesp), São Paulo, SP, Brazil.

3. Faculdade de Ciências Médicas da Santa Casa de São Paulo, São Paulo, SP, Brazil.

4. Hospital Santa Marcelina, São Paulo, SP, Brazil.

5. Quanta, Curitiba, PR, Brazil.

6. Laboratório Bacchi, Botucatu, SP, Brazil.

7. Evidências - Kantar Health, São Paulo, SP, Brazil.

8. Takeda Pharma Brazil, São Paulo, SP, Brazil.

Funding: This study was supported by Takeda Pharmaceutical, São Paulo, Brazil.

Reprint requests and correspondence should be addressed to: Tânia Barreto. Rua Estilo Barroco, 721, São Paulo, SP, Brazil. CEP: 04709-011. Telephone: (+55 11) 5188-4508. E-mail: tania.barreto@takeda.com

Declaration of interest: Tânia Barreto is currently a Takeda employee. Luciana Fanti is currently an Evidências-Kantar Health consultant. Carlos Bacchi is the medical director of Laboratório de Patologia Bacchi. Guilherme Perini has a first-degree relative in Merck and a financial relationship with Takeda and Janssen. Valeria Buccheri presents lectures for Takeda. The other authors declared no conflicts of interest.

Acknowledgments: The authors are grateful to Eloisa S. Moreira, from Evidencias - Kantar Health, for collaboration in the development of the review.

Authors' contributions: Guilherme Fleury Perini, Adriana Damasco Penna, Talita Bueno da Silveira, Ricardo De Sa Bigni, Valeria Buccheri, Juliano Cerci, Carlos Bacchi, Flavia Fernandes Pimenta, Luciana Fanti and Tania Barreto have participated in the literature review and manuscript development.
} 


\section{Hodgkin's lymphoma: Do we still have remaining unmet medical needs?}

Hodgkin's lymphoma $(\mathrm{HL})$ is a B-cell malignancy separated into two major subtypes: Nodular Lymphocyte Predominant (NLP) and Classical Hodgkin Lymphoma. (Kuppers et al. 2012) NLP is characterized by the presence of lymphocytepredominant cells (known as popcorn cells), whereas $\mathrm{CHL}$ which accounts for $95 \%$ of the cases - shows the presence of characteristic neoplastic cells, known as Reed-Sternberg cells and Hodgkin cells, respectively. (Kuppers et al. 2012) In both diseases, the neoplastic cells are scattered in a background of non-neoplastic cells that differ in each subtype. HL affects mainly the lymph nodes, but extra-lymphatic organs such as bone marrow, liver and lungs can also be involved. (Kuppers et al. 2012)

While the cause of $\mathrm{HL}$ remains unknown, familial history, viral infections and immune suppression are known to increase the risk of the disease. A classical bimodal distribution is observed, with a first peak of incidence in the third decade of life and a second peak after the age of 50 . The US National Cancer Institute estimates 8,260 new cases of HL in 2017, representing $0.5 \%$ of all new cancer cases. The last prevalence data estimated 204,065 people living with the disease in the US during the year of 2014 (National Cancer Institute 2017). In Brazil, there are few epidemiologic data; the National Institute of Cancer (Instituto Nacional do Câncer José de Alencar Gomes da Silva (INCA) 2018) estimated 2,530 new cases in 2018, affecting 1,480 men and 1,050 women.

$\mathrm{HL}$ has been a field for extensive research and clinical improvements over the last several years and is currently considered to be a cancer with a high probability of control and cure. In fact, the National Cancer Institute shows that the percentage of HL patients surviving 5 years, between 2007 and 2013, was 86.4\% -including patients with advanced disease at presentation (National Cancer Institute 2017) —and shows that currently more than $80 \%$ of all newly diagnosed $\mathrm{HL}$ patients aged 60 years or less are likely to be cured following front-line therapy consisting of multi-agent chemotherapy and radiotherapy. (Ansell Stephen 2012, Borchmann et al. 2012)

However, despite a high likelihood of success with frontline treatment, approximately $5 \%$ to $10 \%$ of cases of $\mathrm{HL}$ may be refractory to the initial chemotherapy or radiation therapy and approximately 10\% to 30\% may experience relapse after a complete initial response to treatment. (Horning 2000, Diehl et al. 2001) Both groups represent a challenging population to treat, with less well-defined prognostic models and poor therapeutic results. (Collins et al. 2014) Primary resistance is generally considered to be of poor prognosis; many patients do not undergo autologous stem cell transplantation (ASCT) due to rapidly progressive disease, poor performance status or other comorbidities, and until recently, virtually no patient
(0-8\%) survived more than 8 years using conventional chemotherapy alone. (Longo et al. 1992, Bonfante et al. 1997, Josting et al. 2000) Relapsed disease is also associated with worse prognosis in the long term, especially if relapse occurs early following response to first-line therapy. A large retrospective analysis performed by the German Hodgkin Study Group (GHSG) identified that failure to attain a temporary remission on first-line treatment in addition to the low-performance status at the time of progression and age above 50 years predicted poor 5-year freedom from second failure (FF2F). (Josting et al. 2000) Other studies also showed that relapsing within 12 months of first-line therapy leads to worse prognosis (Brice et al. 1997, Wheeler et al. 1997, Sureda et al. 2001) compared to an $80 \%$ chance of a second remission for patients with a late relapse. (Longo et al. 1992)

In general, the treatment of R/R HL starts with salvage chemotherapy, followed by HDT and ASCT. Nevertheless, long-term results are still suboptimal even with this approach: approximately 50\% of HL patients relapse after ASCT (Sureda et al. 2005, Majhail et al. 2006) and their prognosis is generally poor, with a median survival of 25 months (Moskowitz et al. 2009), with only a minority or no patients being cured. (Arai et al. 2013, Martinez et al. 2013). To manage this unmet medical need, new therapeutic approaches have been implemented in recent years, with the development of brentuximab vedotin and checkpoint inhibitors. Most of these innovative therapies have recently been approved for $\mathrm{HL}$ treatment and have been implemented in international guidelines.

\section{Guidelines for Treatment of Relapsing/ Refractory Hodgkin Lymphoma}

The 2017 edition of the NCCN guidelines (National Comprehensive Cancer Network (NCCN) 2017) recommends that patients with primary refractory or relapsed disease should start second-line systemic therapy, which includes several cytotoxic multidrug regimens; patients should be then re-staged and referred to HDT and ASCT if responding; treatment with brentuximab vedotin for one year as a consolidation therapy after ASCT should be considered, especially for patients with Deauville 4 prior to ASCT. PD-1 blocking antibodies should be used after BV. The European recommendations were last updated in 2014 (Eichenauer et al. 2014); they also recommended HDT and ASCT for patients with R/R disease, and even tandem ASCT for high-risk patients. BV was recommended in patients failing ASCT or at least two lines of previous therapy if they are not candidates to ASCT.

Although international guidelines provide orientation on disease management, there are no current national treatment guidelines in Brazil for the treatment of Hodgkin's lymphoma.

Therefore, we reviewed the clinical information of these innovative therapies for the management of $\mathrm{R} / \mathrm{R} \mathrm{HL}$. 


\section{Brentuximab Vedotin}

Brentuximab vedotin (BV) is an antibody-drug conjugate (ADC) comprising an anti-CD30 antibody conjugated by a protease cleavable linker to the potent antimicrotubule agent, monomethyl auristatin E (MMAE). It selectively binds to the CD30 receptor, a member of the tumor necrosis factor (TNF) superfamily, expressed by malignant RS cells. After binding to the cell surface, the ADC-CD30 complex initiates internalization and trafficking to the lysosomal compartment, releasing MMAE via proteolytic cleavage. Binding of MMAE to tubulin disrupts the microtubule network, induces cell cycle arrest, and results in apoptotic death of the CD30expressing tumor cell. This is a targeted mechanism of action, as the normal CD30 expression is restricted to a relatively small proportion of activated B cells, T cells, and eosinophils. (Younes et al. 2012)

Brentuximab vedotin was originally granted accelerated approval by the Food and Drug Administration (FDA) in August 2011, by the European Medicines Agency (Moskowitz et al.) in 2012, and by the Brazilian Health Authority (ANVISA) in 2015. Brentuximab vedotin is approved in Brazil for $\mathrm{HL}$ patients that are relapsing or refractory after ASCT or at least two prior therapies, when ASCT or polychemotherapy is not an option, and as a consolidation therapy for patients at high risk of relapse or progression after ASCT. It is also approved for the treatment of adult patients with relapsed or refractory systemic anaplastic large cell lymphoma, both in Brazil and in the US. The main trials that investigated $\mathrm{BV}$ in $\mathrm{HL}$ are described below.

\section{Pivotal phase Il study in relapsing/ refractory Hodgkin lymphoma}

Younes and colleagues conducted the pivotal phase II, single-arm study that established the efficacy and safety of BV in patients with relapsed or refractory HL after ASCT. In the study, a total of 102 patients with histologically documented $\mathrm{HL}$, measurable disease $\geq 1.5 \mathrm{~cm}$ by computed tomography (CT), PET (positron emission tomography)-positive disease and performance status of 0 or 1 were included. Overall, the study population comprised of patients with poor prognosis: $71 \%$ had a primary refractory disease and $42 \%$ had a disease that was refractory to the most recent prior therapy. The median number of previous chemotherapy regimens excluding ASCT was 3.5 and the median time to relapse after ASCT was only 6.7 months, with most patients (71\%) having relapsed within a year of ASCT. All 102 patients were treated with at least one infusion of brentuximab vedotin $1.8 \mathrm{mg} / \mathrm{kg}$ every 3 weeks; in the absence of disease progression or prohibitive toxicity, patients received a maximum of 16 cycles. The overall response rate (ORR - primary endpoint) was $75 \%$, with complete remission (Armand et al.) in 34\% of patients. The median time to the objective and to complete response were 5.7 and 12 weeks, respectively. The median duration of response was 6.7 months among all responders, while it was 20.5 months for those who achieved a CR. Responses occurred in different subgroups, including relapsed and primary refractory disease. The median progression-free survival (PFS) for all patients was 5.6 months and for patients who achieved a CR was 21.7 months. Median overall survival (OS) was 22.4 months, with an estimated 1-year survival rate of $89 \%$. These responses seen with BV were superior to responses observed with other single agents. In addition, PFS was longer than the PFS observed with the previous systemic therapies (for patients who received systemic therapy following ASCT). Most adverse events (AEs) associated with BV were grade 1 or 2 and manageable through standard supportive care. They included peripheral sensory neuropathy, nausea, fatigue, neutropenia, and diarrhea. Neuropathy was the most clinically meaningful AE although it was largely reversible and considered to be a class effect of antimicrotubule agents. (Younes et al. 2012)

\section{Follow-up data}

At 5 years, patients from the pivotal phase II study of BV for $\mathrm{R} / \mathrm{R} \mathrm{HL}$ received a median of nine cycles of $\mathrm{BV}$ with an ORR of $72 \%$ and the CR rate of $33 \%$. The median OS and PFS were 40.5 and 9.3 months, respectively, and the estimated 5-year OS and PFS rates were $41 \%$ and $22 \%$. Interestingly, patients who had achieved a complete response with brentuximab $(\mathrm{N}=34)$ had estimated 5-year OS and PFS rates of 64\% and $52 \%$, respectively, without achieving the median OS and PFS. This final analysis showed that 13 patients (38\% of all CR patients) were still on follow-up and remission at study closure; 9 of them in sustained CR without receiving any further anticancer therapy (4 received allogeneic stem cell transplant [allo-SCT]). These patients in long-term remission represent 9\% of the enrolled study population and provide a new perspective on the belief that allo-SCT was the only option for long-term disease control. The most common treatmentrelated AEs were peripheral sensory neuropathy, nausea, fatigue, neutropenia, and diarrhea. Grade 3 or higher AEs occurred in $\geq 5$ percent of patients and included neutropenia, peripheral sensory neuropathy, thrombocytopenia, and anemia. Of the patients who experienced treatment-emergent peripheral neuropathy, 88\% achieved either resolution (73\%) or improvement (14\%) in symptoms. (Chen et al. 2016)

The encouraging experience with $\mathrm{BV}$ in the setting of relapse or refractory disease led to its investigation in the consolidation of remission and prevention of relapse in high-risk patients following ASCT.

\section{Brentuximab vedotin as consolidation following ASCT}

Patients with HL who relapse after ASCT have a poor outcome with very few effective therapeutic options. A study in 
this population showed a median time to progression after the next therapy of only 3.8 months and a median survival after the ASCT of 26 months. (Kewalramani et al. 2003) Patients who underwent a reduced-intensity allogeneic transplant had a treatment-related mortality at 1 year of $20 \%$ and a 2-year OS of 50\%. (Robinson et al. 2002) In fact, no completed randomized trials of maintenance or consolidation therapy after ASCT had been reported and no treatment was approved in this setting, and the standard of care for these patients was observation and best supportive care until disease progression. The unmet need of this population coupled with the success of BV in the relapsed and refractory HL setting raised the prospect of its use in prolonging remission and preventing relapse in high-risk patients following ASCT.

The randomized, placebo-controlled Aethera trial (Moskowitz et al. 2015) investigated the use of BV after ASCT in 329 patients with a high risk of relapse following ASCT. Risk factors for relapse following ASCT were based on data from multiple series of ASCT patients and validated prognostic models and were defined as one of the following: primary refractory $\mathrm{HL}$ (failure to achieve complete remission), early relapsed disease (remission lasting less than 1 year) or the presence of extranodal disease at the start of salvage therapy. However, patients included had to have had at least stable disease after salvage. A standard regimen of BV was started 30-45 days after ASCT. CT scans were used to assess disease progression according to an independent review and the Revised Response Criteria for Malignant Lymphoma.

BV appeared to be well-tolerated in this setting, with nearly $50 \%$ of patients completing 16 cycles of therapy and a toxicity profile that was consistent with previous studies. Peripheral neuropathy was the most common AE, with resolution or improvement in most patients. Consolidation treatment with BV provided a statistically and clinically significant improvement in PFS versus placebo. After a median follow-up of 30 months, PFS was significantly improved in the BV group, with a $43 \%$ reduction in the risk of progression (HR: $0.57 ; 95 \% \mathrm{Cl} 0.40-0.81 ; p=0.0013$ ). Median PFS with brentuximab vedotin was 42.9 months versus 24.1 months in the placebo group, with estimated 2-year rates of PFS of $63 \%$ and $51 \%$, respectively. PFS was consistent across all subgroups. Overall, there was high concordance (87\%) between central and investigator assessments with more progression events being recorded by the investigators (for 13\% of the patients in the placebo group and $4 \%$ of the patients in the BV group). The authors recorded only four PFS events after the 24-month assessment period, encompassing 108 patient-years of follow-up.

There was no difference in OS between treatment groups in the interim analysis, probably due to the high crossover rate of patients in the placebo group. Although more followup data is needed to evaluate long-term survival, the authors consider that many patients who were progression-free at 24 months might be cured, since relapse or progression after ASCT usually happens early (71\% within 1 year and $90 \%$ within 2 years of ASCT). (Majhail et al. 2006, Arai et al. 2013) Lastly, a post-hoc analysis conducted by the authors showed that the PFS and OS benefits with BV increased with an increase in the numbers of risk factors for relapse after ASCT; however, further analysis of risk factors is needed to define better the population that is most likely to benefit from consolidation therapy with BV after ASCT.

At 3 years, in the Aethera trial follow-up, it was observed that the PFS rate was $61 \%$ (95\% Cl 53-68) for the BV arm and $43 \%$ (95\% Cl 36-51) for the placebo group. The PFS was similar to the one observed in the 2-year follow up, with a HR of 0.58 (95\% Cl 0.41-0.82). (Sweetenham 2016) Also, another study showed that BV presents a stronger impact in $\mathrm{HL}$ patients with more than 2 risk factors. (C. Moskowitz 2016)

\section{Retreatment with Brentuximab Vedotin}

Retreatment with BV in patients with $\mathrm{HL}(\mathrm{n}=20)$ who had achieved CR or PR during initial BV therapy and subsequently relapsed was associated with a high overall objective response rate, with the same rate of CR and PR (both 30\%) and $20 \%$ of patients experiencing stable disease as a best response. The median time between the last dose of $\mathrm{BV}$ given during the initial study and the first dose of retreatment was 11.4 months (range 4-45 months). No patients with $\mathrm{HL}$ were retreated with $B V$ more than once (permitted if required). At this time point, the median OS had not yet been reached. (Bartlett et al. 2014)

\section{Checkpoint Inhibitors}

Over the past several years, a greater understanding of the regulatory pathways of the immune system and the mechanisms that allow tumor escape, including tumor augmentation of ligands to the $T$ cell-programmed cell death 1 (PD-1) receptor known as PD-L1 and PD-L2 have led to the development of new immunotherapeutic options to treat solid tumors. This approach is especially interesting to treat hematologic malignancies based on the efficacy of immunologic approaches such as allogeneic transplantation, immunomodulatory drugs and adoptive cellular therapies (Tai et al. 2005, Maus et al. 2014) and the expression of PD-L1 and PD-L2. (Bryan and Gordon 2015) Specifically in HL, other factors provide further support to immunologic treatment such as the high expression of the immunoregulatory glycanbinding protein and galectin-1 (resulting in a type 2 T-helper cell and T-regulatory cell skewed tumor micro-environment), immunoregulatory gene alterations such as amplification at the 9p24.1 locus (resulting in increased JAK-STAT signaling and high expression of PD-L1 and PD-L2 on the tumor cell 
surface) (Green et al. 2010) and the presence of latent Epstein-Barr virus infection in around $40 \%$ of $\mathrm{HL}$ cases, which is also thought to contribute to high expression of PD-L1 and PD-L2. (Paydas et al. 2015) Therefore, tumor-immune evasion through PD-1 augmentation appears to play a major role in the oncogenesis of $\mathrm{HL}$ and has consequently led to considerable interest in immune checkpoint inhibition using anti-PD-1 and anti-PD-L1 immunotherapy. Increasing data have been presented in the last few years about the use of checkpoint inhibitors, mainly on the anti-PD1 monoclonal antibodies nivolumab and pembrolizumab in the treatment of $R / R H L$.

\section{Nivolumab}

Nivolumab is a fully human monoclonal lgG4 antibody directed against PD-1. It has been approved by the FDA for HL adult patients who relapsed or progressed after hematologic stem cell transplantation (HSCT) and BV, or after at least 3 lines of systemic therapy, including HSCT. It has also been approved by the EMA for patients with relapsed or refractory $\mathrm{HL}$ after ASCT and BV. Nivolumab was approved by ANVISA in October 2017 for the treatment of patients with relapsing or refractory HL after ASCT followed by therapy with brentuximab vedotin.

\section{Phase II: Nivolumab after failure of ASCT and brentuximab vedotin}

The phase II CheckMate 205 study was a multi-cohort study evaluating nivolumab in $\mathrm{HL}$; data from the cohort of patients with prior failure of both ASCT and subsequent BV has been published (Timmerman et al. 2016). Patients were included regardless of their PD-L1 status and received nivolumab 3mg/ $\mathrm{kg}$ every 2 weeks. They were required to have received previous BV but were not required to be refractory to it. The median number of previous therapies was four. The median time between the most recent $\mathrm{BV}$ treatment and the first dose of nivolumab was 0.7 years, and the median time between HDT / ASCT and the first dose of nivolumab was 3.4 years.

At the time of analysis, $64 \%$ of patients remained on treatment and the median number of nivolumab doses received was 17 . The primary outcome was ORR assessed by a central, independent review group. At a median follow-up of 8.9 months, $66.3 \%$ of the 80 patients achieved an objective response, which included CR in seven (9\%) patients and PR in 46 (58\%) patients. Overall, responses were quick, with a median time to first objective response of 2.1 months, and profound, with tumor reductions of at least $50 \%$ from baseline in all but one patient. The median duration of objective response was 7.8 months. Similar to what has been seen in solid tumors, there were some atypical response patterns to PD-1 blockade, including the appearance of new lesions followed by negative PET scan and responses after initial progression. Of note, response to nivolumab was reported in more than twothirds of patients who had not responded to the most recent BV treatment before trial enrolment. At 6 months, the PFS rate was $76.9 \%$ and OS rate was $98.7 \%$. At 12 months, median PFS was 10.0 months ( $95 \%$ Cl 8.41-not reached). Some patients discontinued treatment with nivolumab and were referred to stem cell transplant (5 allo-SCT and one ASCT); one of them was in CR by the time of referral, 2 were in PR and one in stable disease. All patients who had transplantation after nivolumab treatment were alive at the time of analysis; however, there is a risk of complication in patients receiving alloSCT, and they should be monitored for complications such as hyperacute graft-versus host-disease (GVHD), grade 3-4 acute GVHD, steroid-requiring febrile syndrome, hepatic veno-occlusive disease, and other immune-mediated adverse reactions as four of five patients died from complications of allo-SCT in the phase 1 study. Overall, the most common drug-related AES ( $\geq 15 \%$ of patients) included fatigue (25\%), infusion-related reaction (20\%), and rash (16\%), and the most common drug-related grade 3 or 4 AEs were neutropenia $(5 \%)$ and increased lipase concentrations (5\%). AEs of special interest, which are possibly inflammatory in nature and due to the enhancement of the immunologic activity following PD1 blockade were skin abnormalities (41\%); gastrointestinal abnormalities (26\%); hypersensitivity or infusion-related reaction (21\%); and endocrine (18\%), hepatic (10\%), renal (5\%), and pulmonary (1\%) events. Pneumonitis was reported in two (3\%) patients (one grade 2 and one grade 3 ) between the first dose and 35 days after the last dose; both cases were judged to be drug-related and both resolved with corticosteroid treatment. Most select AEs of special interest reported were of grades 1 or 2 , and most were considered by the investigators to be drug-related.

\section{Follow-up data}

Follow-up data from CheckMate 205 has recently been presented. (Timmerman et al. 2016) For the cohort of patients who failed ASCT and BV, 43 patients (54\%) remained on therapy after a median follow-up of 15.4 months. The ORR was $68 \%$, with a CR rate of $8 \%$ and a PR rate of $60 \%$. The median duration of response was prolonged to 13.1 months $(95 \% \mathrm{Cl}$, 8.7 months - not reached; range, 0.0-14.2 months) and the median duration of CR was not reached. Median PFS was 14.8 months; 12 -month PFS was $54.6 \%$ and 12 -month overall survival (OS) was $94.9 \%$, with median OS not reached. The most common reasons for discontinuation were disease progression (24\%), allogeneic SCT (9\%) and AEs (6\%). The most common drug-related AEs were fatigue (28\%), infusion reaction (20\%), arthralgia (15\%), and rash (15\%). Twenty-nine percent of patients had Grade 3-4 drug-related AEs; the most common were increased lipase (8\%), neutropenia (5\%), and increased aspartate aminotransferase (4\%). 


\section{Pembrolizumab}

Pembrolizumab is a humanized immunoglobulin G-1 kappa monoclonal antibody targeting the PD-1 receptor, which has also demonstrated a significant effect in HL. The FDA approved it for the treatment of adult and pediatric patients with refractory disease or who have relapsed after 3 or more prior lines of therapy. EMA has approved the drug for adult patients with R/R HL who have failed autologous ASCT and $B V$, or who are transplant-ineligible and have failed BV. Until the approval of this article for publication, pembrolizumab had not been approved for treatment of HL in Brazil.

\section{Phase II: Pembrolizumab in different cohorts of relapsed/refractory patients}

Keynote 087 was a single-arm phase II study which investigated pembrolizumab at the fixed dose of $200 \mathrm{mg}$ every 3 weeks in 210 heavily pretreated patients with R/R HL. Patients were grouped in cohorts based on disease progression: if they had progressed after ASCT and subsequent BV (cohort 1), after salvage chemotherapy and BV (ineligible to ASCT_cohort 2) or after ASCT but without BV (cohort 3). The primary end points were ORR by central review and safety. The population included $35 \%$ of patients with primary refractory disease and $14 \%$ whose disease was chemo-refractory to all prior regimens; $61 \%$ had undergone prior ASCT and $83 \%$ had received prior BV at some point, including $41.7 \%$ of patients in cohort 3, who had received BV treatment before ASCT. Across all cohorts, $90 \%$ of the patients had some level of decrease in the tumor burden. Most responses were reported at the first assessment and ORR was 69\%, with a CR rate of $22.4 \%$. An analysis by cohort was prespecified; although the patients had received different prior treatments, their response rates were similar (from 64\% for cohort 2 to $73.9 \%$ for cohort 1), as well as the rates of CR (20\% to $25 \%$ ). The median duration of response was not reached in any of the cohorts by the time of the analysis. At 6 months, the OS rate was 99.5\% (median OS not reached) and the PFS rate was $72.4 \%$. Due to the short follow-up, more accurate estimations of OS and PFS, as well as durability of response, are needed.

The safety profile was largely consistent with previous studies, with the most common treatment-related AEs being $11 \%$, hypothyroidism $10.5 \%$, diarrhea $6.7 \%$, fatigue $6.7 \%$, headache $6.2 \%$, rash $6.2 \%$ and nausea $5.7 \%$. Importantly, 14 patients in this study went on to receive a stem cell transplant, including 10 patients undergoing allogeneic SCT; one of these patients died due to graft-versus-host disease. Similar to nivolumab, it is recommended to monitor patients for complications of allo-SCT after treatment with pembrolizumab, including hepatic veno-occlusive disease, grade 3-4 acute GVHD, including hyperacute GVHD, steroid-requiring febrile syndrome, and other immune-mediated adverse reactions. (Chen et al. 2017).

\section{Conclusion}

$\mathrm{HL}$ is currently considered a cancer with a high probability of cure with front-line therapy based on multiagent chemotherapy and radiotherapy. However, up to $30 \%$ of patients will be refractory to frontline therapy or present with disease relapse following initial response to therapy. Salvage chemotherapy followed by high dose therapy and ASCT is the standard of care for this population, but outcomes are inferior to front-line therapy, with approximately half of the patients relapsing after ASCT. New therapies for R/R HL such as brentuximab vedotin and checkpoint inhibitors provide new therapeutic options for patients, with good tolerability, high response rates and promising survival results, even after failure of ASCT. For patients with a high risk of relapse following ASCT, the positive data of brentuximab vedotin in the consolidation setting also provides a new standard of care in the management of the disease.

\section{Acknowledgments}

The authors are grateful to Eloisa S. Moreira, from Evidencias - Kantar Health, for collaboration in the development of the review.

\section{References}

Ansell Stephen, M. (2012). "Hodgkin lymphoma: 2012 update on diagnosis, risk-stratification, and management." American Journal of Hematology 87(12): 1096-1103.

Arai, S., M. Fanale, S. DeVos, A. Engert, T. Illidge, P. Borchmann, A. Younes, F. Morschhauser, A. McMillan and S. J. Horning (2013). "Defining a Hodgkin lymphoma population for novel therapeutics after relapse from autologous hematopoietic cell transplant." Leuk Lymphoma 54(11): 2531-2533.

Armand, P., M. A. Shipp, V. Ribrag, J.-M. Michot, P. L. Zinzani, J. Kuruvilla, Y. Zhu, A. D. Ricart, A. Balakumaran and C. H. Moskowitz (2016). "Pembrolizumab in Patients with Classical Hodgkin Lymphoma after Brentuximab Vedotin Failure: Long-term Efficacy from the Phase $1 \mathrm{~b}$ Keynote-013 Study." Blood 128(22): 1108.

Bartlett, N. L., R. Chen, M. A. Fanale, P. Brice, A. Gopal, S. E. Smith, R. Advani, J. V. Matous, R. Ramchandren, J. D. Rosenblatt, D. Huebner, P. Levine, L. Grove and A. Forero-Torres (2014). "Retreatment with brentuximab vedotin in patients with CD30-positive hematologic malignancies." J Hematol Oncol 7: 24.

Bonfante, V., A. Santoro, S. Viviani, L. Devizzi, M. Balzarotti, F. Soncini, M. Zanini, P. Valagussa and G. Bonadonna (1997). "Outcome of patients with Hodgkin's disease failing after primary MOPP-ABVD." J Clin Oncol 15(2): 528-534.

Borchmann, P., D. A. Eichenauer and A. Engert (2012). "State of the art in the treatment of Hodgkin lymphoma." Nat Rev Clin Oncol 9(8): 450-459.

Brice, P., R. Bouabdallah, P. Moreau, M. Divine, M. Andre, M. Aoudjane, J. Fleury, B. Anglaret, A. Baruchel, L. Sensebe and P. Colombat (1997). "Prognostic factors for survival after high-dose therapy and autologous stem cell transplantation for patients with relapsing Hodgkin's disease: Analysis of 280 patients from the French registry. Societe Francaise de Greffe de Moelle." Bone Marrow Transplant 20(1): 21-26. 
Bryan, L. J. and L. I. Gordon (2015). "Blocking tumor escape in hematologic malignancies: The anti-PD-1 strategy." Blood Rev 29(1): 25-32.

C. Moskowitz, J. S., A. Chen, E. Ayala, T. Masszi, J. Holowiecki, P. Stiff, A. Carella, S. Viviani, V. Bachanova, A. Sureda, D. Huebner, S.Y. Lee, N. Hunder, L. Thomas, M. Uttarwar, J. Walewski (2016). Brentuximab Vedotin After Autologous Stem Cell Transplant Yields the Strongest Benefit in Hodgkin Lymphoma Patients With $\geq 2$ Risk Factors: Results of a Multivariate Analysis International Symposium on Hodgkin Lymphoma. Colonia: abstract 44.

Chen, R., A. K. Gopal, S. E. Smith, S. M. Ansell, J. D. Rosenblatt, K. J. Savage, J. M. Connors, A. Engert, E. K. Larsen, D. Huebner, A. Fong and A. Younes (2016). "Five-year survival and durability results of brentuximab vedotin in patients with relapsed or refractory Hodgkin lymphoma." Blood 128(12): 1562-1566.

Chen, R., P. L. Zinzani, M. A. Fanale, P. Armand, N. A. Johnson, P. Brice, J. Radford, V. Ribrag, D. Molin, T. P. Vassilakopoulos, A. Tomita, B. von Tresckow, M. A. Shipp, Y. Zhang, A. D. Ricart, A. Balakumaran and C. H. Moskowitz (2017). "Phase II Study of the Efficacy and Safety of Pembrolizumab for Relapsed/ Refractory Classic Hodgkin Lymphoma." J Clin Oncol 35(19): 2125-2132.

Collins, G. P., A. N. Parker, C. Pocock, I. Kayani, A. Sureda, T. Illidge, K. Ardeshna, D. C. Linch and K. S. Peggs (2014). "Guideline on the management of primary resistant and relapsed classical Hodgkin lymphoma." Br J Haematol 164(1): 39-52.

Diehl, V., P. Mauch and N. Harris (2001). Hodgkin's disease. Principles and Practice of Oncology. H. S. In De Vita VT, Rosenberg SA. Philadelphia: Lippincott Williams \& Wilkins. 6th ed: 2339-2386.

Eichenauer, D. A., A. Engert, M. Andre, M. Federico, T. Illidge, M. Hutchings and M. Ladetto (2014). "Hodgkin's lymphoma: ESMO Clinical Practice Guidelines for diagnosis, treatment and follow-up." Ann Oncol 25 Suppl 3: iii70-75.

Green, M. R., S. Monti, S. J. Rodig, P. Juszczynski, T. Currie, E. O'Donnell, B. Chapuy, K. Takeyama, D. Neuberg, T. R. Golub, J. L. Kutok and M. A. Shipp (2010). "Integrative analysis reveals selective 9p24.1 amplification, increased PD-1 ligand expression, and further induction via JAK2 in nodular sclerosing Hodgkin lymphoma and primary mediastinal large B-cell lymphoma." Blood 116(17): 3268-3277.

Horning, S. (2000). Hodgkin's disease. Textbook of Medical Oncology. London: Martin Dunitz Publishers: 461-474.

Instituto Nacional do Câncer José de Alencar Gomes da Silva (INCA). (2018). Retrieved Jun 2018, 2018, from http://www2.inca.gov.br/wps/wcm/ connect/tiposdecancer/site/home/linfoma_hodgkin.

Josting, A., U. Rueffer, J. Franklin, M. Sieber, V. Diehl and A. Engert (2000). "Prognostic factors and treatment outcome in primary progressive Hodgkin lymphoma: A report from the German Hodgkin Lymphoma Study Group." Blood 96(4): 1280-1286.

Kewalramani, T., S. D. Nimer, A. D. Zelenetz, S. Malhotra, J. Qin, J. Yahalom and C. H. Moskowitz (2003). "Progressive disease following autologous transplantation in patients with chemosensitive relapsed or primary refractory Hodgkin's disease or aggressive non-Hodgkin's lymphoma." Bone Marrow Transplant 32(7): 673-679.

Kuppers, R., A. Engert and M. L. Hansmann (2012). "Hodgkin lymphoma." J Clin Invest 122(10): 3439-3447.

Longo, D. L., P. L. Duffey, R. C. Young, S. M. Hubbard, D. C. Ihde, E. Glatstein, J. C. Phares, E. S. Jaffe, W. J. Urba and V. T. DeVita, Jr. (1992). "Conventional-dose salvage combination chemotherapy in patients relapsing with Hodgkin's disease after combination chemotherapy: The low probability for cure." J Clin Oncol 10(2): 210-218.

Majhail, N. S., D. J. Weisdorf, T. E. Defor, J. S. Miller, P. B. McGlave, A. Slungaard, M. Arora, N. K. Ramsay, P. J. Orchard, M. L. MacMillan and L. J. Burns (2006). "Long-term results of autologous stem cell transplantation for primary refractory or relapsed Hodgkin's lymphoma." Biol Blood Marrow Transplant 12(10): 1065-1072.
Martinez, C., C. Canals, B. Sarina, E. P. Alessandrino, D. Karakasis, A. Pulsoni, S. Sica, M. Trneny, J. A. Snowden, E. Kanfer, N. Milpied, A. Bosi, S. Guidi, C. A. de Souza, R. Willemze, R. Arranz, L. Jebavy, A. Hellmann, D. Sibon, R. Oneto, J. J. Luan, P. Dreger, L. Castagna and A. Sureda (2013). "Identification of prognostic factors predicting outcome in Hodgkin's lymphoma patients relapsing after autologous stem cell transplantation." Ann Oncol 24(9): 2430-2434.

Maus, M. V., S. A. Grupp, D. L. Porter and C. H. June (2014). "Antibody-modified T cells: CARs take the front seat for hematologic malignancies." Blood 123(17): 2625-2635.

Moskowitz, A. J., M. A. Perales, T. Kewalramani, J. Yahalom, H. Castro-Malaspina, Z. Zhang, J. Vanak, A. D. Zelenetz and C. H. Moskowitz (2009). "Outcomes for patients who fail high dose chemoradiotherapy and autologous stem cell rescue for relapsed and primary refractory Hodgkin lymphoma." Br J Haematol 146(2): 158-163.

Moskowitz, C. H., A. Nademanee, T. Masszi, E. Agura, J. Holowiecki, M. H. Abidi, A. I. Chen, P. Stiff, A. M. Gianni, A. Carella, D. Osmanov, V. Bachanova, J. Sweetenham, A. Sureda, D. Huebner, E. L. Sievers, A. Chi, E. K. Larsen, N. N. Hunder and J. Walewski (2015). "Brentuximab vedotin as consolidation therapy after autologous stem-cell transplantation in patients with Hodgkin's lymphoma at risk of relapse or progression (AETHERA): A randomised, double-blind, placebo-controlled, phase 3 trial." Lancet 385(9980): 1853-1862.

National Cancer Institute. (2017). "Cancer Stat Facts: Hodgkin Lymphoma." Retrieved Jun 2017, 2017, from https://seer.cancer.gov/statfacts/html/ hodg.html.

National Comprehensive Cancer Network (NCCN). (2017). "Clinical Practice Guidelines in Oncology", Jun 2017, from https://www.ncen.org/ professionals/physician_gls/default.aspx.

Paydas, S., E. Bağır, G. Seydaoglu, V. Ercolak and M. Ergin (2015). "Programmed death-1 (PD-1), programmed death-ligand 1 (PD-L1), and EBV-encoded RNA (EBER) expression in Hodgkin lymphoma." Annals of hematology 94(9): 1545-1552.

Robinson, S. P., A. H. Goldstone, S. Mackinnon, A. Carella, N. Russell, C. R. de Elvira, G. Taghipour and N. Schmitz (2002). "Chemoresistant or aggressive lymphoma predicts for a poor outcome following reducedintensity allogeneic progenitor cell transplantation: An analysis from the Lymphoma Working Party of the European Group for Blood and Bone Marrow Transplantation." Blood 100(13): 4310-4316.

Sureda, A., R. Arranz, A. Iriondo, E. Carreras, J. J. Lahuerta, J. Garcia-Conde, I. Jarque, M. D. Caballero, C. Ferra, A. Lopez, J. Garcia-Larana, R. Cabrera, D. Carrera, M. D. Ruiz-Romero, A. Leon, J. Rifon, J. Diaz-Mediavilla, R. Mataix, M. Morey, J. M. Moraleda, A. Altes, A. Lopez-Guillermo, J. de la Serna, J. M. Fernandez-Ranada, J. Sierra and E. Conde (2001). "Autologous stem-cell transplantation for Hodgkin's disease: Results and prognostic factors in 494 patients from the Grupo Espanol de Linfomas/Transplante Autologo de Medula Osea Spanish Cooperative Group." J Clin Oncol 19(5): 1395-1404.

Sureda, A., M. Constans, A. Iriondo, R. Arranz, M. D. Caballero, M. J. Vidal, J. Petit, A. Lopez, J. J. Lahuerta, E. Carreras, J. Garcia-Conde, J. Garcia-Larana, R. Cabrera, I. Jarque, D. Carrera, J. C. Garcia-Ruiz, M. J. Pascual, J. Rifon, J. M. Moraleda, K. Perez-Equiza, C. Albo, J. Diaz-Mediavilla, A. Torres, P. Torres, J. Besalduch, J. Marin, M. V. Mateos, J. M. Fernandez-Ranada, J. Sierra and E. Conde (2005). "Prognostic factors affecting long-term outcome after stem cell transplantation in Hodgkin's lymphoma autografted after a first relapse." Ann Oncol 16(4): 625-633.

Sweetenham, J. W. E. A. (2016). "Updated Efficacy and Safety Data from the AETHERA Trial of Consolidation with Brentuximab Vedotin after Autologous Stem Cell Transplant (ASCT) in Hodgkin Lymphoma Patients at High Risk of Relapse." Biology of Blood and Marrow Transplantation Volume 22 (Issue 3): S36-S37. 
Tai, Y. T., X. Li, X. Tong, D. Santos, T. Otsuki, L. Catley, O. Tournilhac, K. Podar, T. Hideshima, R. Schlossman, P. Richardson, N. C. Munshi, M. Luqman and K. C. Anderson (2005). "Human anti-CD40 antagonist antibody triggers significant antitumor activity against human multiple myeloma." Cancer Res 65(13): 5898-5906.

Timmerman, J. M., A. Engert, A. Younes, A. Santoro, P. Armand, M. A. Fanale, G. P. Collins, V. Ratanatharathorn, J. Kuruvilla, J. B. Cohen, K. J. Savage, M. Trneny, J. P. De Boer, M. A. Shipp, S. J. Rodig, K. Kato, A. Sumbul and S. Ansell (2016). "Checkmate 205 Update with Minimum 12-Month Follow up: A Phase 2 Study of Nivolumab in Patients with Relapsed/Refractory Classical Hodgkin Lymphoma." Blood 128(22): 1110.

Wheeler, C., C. Eickhoff, A. Elias, J. Ibrahim, L. Ayash, M. McCauley, P. Mauch, G. Schwartz, J. P. Eder, R. Mazanet, J. Ferrara, I. J. Rimm, E. Guinan, B. Bierer, G. Gilliland, W. H. Churchill, K. Ault, S. Parsons, K. Antman, L. Schnipper, I. Tepler, L. Gaynes, E. Frei, M. Kadin and J. Antin (1997). "High-dose cyclophosphamide, carmustine, and etoposide with autologous transplantation in Hodgkin's disease: A prognostic model for treatment outcomes." Biology of blood and marrow transplantation: Journal of the American Society for Blood and Marrow Transplantation 3(2): 98-106.
Younes, A., A. K. Gopal, S. E. Smith, S. M. Ansell, J. D. Rosenblatt, K. J. Savage, R. Ramchandren, N. L. Bartlett, B. D. Cheson, S. de Vos, A. Forero-Torres, C. H. Moskowitz, J. M. Connors, A. Engert, E. K. Larsen, D. A. Kennedy, E. L. Sievers and R. Chen (2012). "Results of a pivotal phase II study of brentuximab vedotin for patients with relapsed or refractory Hodgkin's lymphoma." J Clin Oncol 30(18): 2183-2189. 